\title{
Another new approach to the small Ree groups
}

\author{
Robert A. Wilson
}

Abstract. A new elementary construction of the small Ree groups is described.

Mathematics Subject Classification (2000). 20D06.

Keywords. Groups of Lie type, simple groups, Ree groups.

\section{Introduction}

The Ree groups of characteristic 3 were originally discovered in about 1960 by Rimhak Ree [3]. They were constructed as the fixed points of certain automorphisms of order 2 of the Dickson-Chevalley groups $G_{2}\left(3^{2 n+1}\right)$, acting on the Lie algebra of dimension 14 . As this Lie algebra has a 7-dimensional ideal, it is possible to describe the Ree groups acting on a 7-dimensional vector space, or a 6-dimensional projective space. This is how Tits [5] described the group, in effect giving an independent existence proof, although the substantial calculations required were not published.

In [7] I presented a new construction of the Ree groups of characteristic 3, including complete calculations, using a moderately complicated object which I called the octopus algebra. Subsequently I have found a way of simplifying the construction substantially, which among other benefits removes a great deal of the calculations from the proofs. This new construction is presented here in the hope that in time it (or something very like it) will come to be regarded as the standard construction.

A similar construction of the Ree groups of characteristic 2 is described in [8], which provides the first genuinely elementary proof of existence of these groups, and is independent of Ree's original proof [4]. The analogue for the Suzuki groups is even simpler [9], and provides a complete, elementary, and constructive proof of existence (and simplicity) of these groups in about three pages. 


\section{Definitions}

First we set up the octonion algebra. Let $n$ be a non-negative integer, let $F=\mathbb{F}_{q}$ be the field of order $q=3^{2 n+1}$, and let $W$ be a 7-dimensional vector space over $F$ with basis $\left\{e_{i} \mid-3 \leq i \leq 3\right\}$. Let $b$ be the symmetric bilinear form defined on $W$ by $b\left(e_{i}, e_{-i}\right)=1$ and all other $b\left(e_{i}, e_{j}\right)=0$. Let $t$ be the alternating trilinear form on $W$ defined by $t\left(e_{i}, e_{j}, e_{k}\right)=0$ unless $i+j+k=0$, and $t\left(e_{i}, e_{j}, e_{k}\right)=1$ when $(i, j, k)$ is a cyclic permutation of one of the triples $(0,1,-1),(0,2,-2),(0,-3,3)$, $(1,2,-3)$ or $(-1,-2,3)$. [Recall that alternating means $t(u, v, w)=t(v, w, u)$ and $t(u, u, v)=0$, so $t(v, u, w)=-t(u, v, w)$.] There is now a natural product $*$ on $W$ defined by $t(u, v, w)=b(u * v, w)$. We call this the octonion product as it can be identified with the product on the pure imaginary part of a split octonion algebra. On the basis vectors, the non-zero products are given by $e_{i} * e_{j}=e_{k},-e_{k}$ according as $t\left(e_{i}, e_{j}, e_{-k}\right)=1,-1$. In particular the product is graded in the sense that $e_{i} * e_{j} \in\left\langle e_{i+j}\right\rangle$ (where this is interpreted as zero if $i+j$ is not in the range -3 to 3 ). To assist with calculations, I include the multiplication table for this product here. In this table, blank entries denote zero.

\begin{tabular}{c|ccccccc|}
$*$ & $e_{-3}$ & $e_{-2}$ & $e_{-1}$ & $e_{0}$ & $e_{1}$ & $e_{2}$ & $e_{3}$ \\
\hline$e_{-3}$ & & & & $-e_{-3}$ & $e_{-2}$ & $-e_{-1}$ & $e_{0}$ \\
$e_{-2}$ & & & $-e_{-3}$ & $e_{-2}$ & & $-e_{0}$ & $e_{1}$ \\
$e_{-1}$ & & $e_{-3}$ & & $e_{-1}$ & $-e_{0}$ & & $-e_{2}$ \\
$e_{0}$ & $e_{-3}$ & $-e_{-2}$ & $-e_{-1}$ & & $e_{1}$ & $e_{2}$ & $-e_{3}$ \\
$e_{1}$ & $-e_{-2}$ & & $e_{0}$ & $-e_{1}$ & & $e_{3}$ & \\
$e_{2}$ & $e_{-1}$ & $e_{0}$ & & $-e_{2}$ & $-e_{3}$ & & \\
$e_{3}$ & $-e_{0}$ & $-e_{1}$ & $e_{2}$ & $e_{3}$ & & & \\
\hline
\end{tabular}

The new ingredient of the construction is an alternating bullet product $u \bullet v$ defined whenever $u$ and $v$ are isotropic vectors with $u * v=0$, satisfying the twisted linearity law

$$
(u+\lambda v) \bullet w=u \bullet w+\lambda^{3^{n}}(v \bullet w) .
$$

In order to see that $\bullet$ is well-defined, it is easiest to reinterpret the octonion product as a linear map $\kappa$ from $W \wedge W$ to $W$, and then reinterpret $\bullet$ as a map $\pi$ from the kernel of $\kappa$ to $W$. The twisted linearity conditions imply that it is necessary and sufficient to define $\pi$ on a basis of ker $\kappa$, which has dimension 14 . The equations below do exactly this. The bullet product is invariant under the symmetry $r: e_{0} \mapsto-e_{0}, e_{i} \mapsto e_{-i}$ for $i \neq 0$, and is defined by the rules

$$
\begin{aligned}
e_{3} \bullet e_{2} & =e_{3} \\
e_{1} \bullet e_{3} & =e_{2} \\
e_{2} \bullet e_{-1} & =e_{1} \\
e_{-1} \bullet e_{1}+e_{2} \bullet e_{-2} & =e_{0}
\end{aligned}
$$

[The last line of the display is shorthand for $\left(e_{-1}+e_{-2}\right) \bullet\left(e_{1}-e_{2}\right)=e_{1}+e_{0}+e_{-1}$, cancelling out the cross terms, since $e_{-1} \bullet e_{1}$ and $e_{-2} \bullet e_{2}$ are not themselves 
defined.] All 'other' products are zero, in the sense that

$$
\begin{aligned}
e_{0} \bullet e_{1}+e_{3} \bullet e_{-2} & =0 \\
e_{0} \bullet e_{2}+e_{-1} \bullet e_{3} & =0 \\
e_{0} \bullet e_{3}+e_{1} \bullet e_{2} & =0 \\
e_{-3} \bullet e_{3}+e_{2} \bullet e_{-2}+e_{1} \bullet e_{-1} & =0
\end{aligned}
$$

These equations in (strictly) undefined products are equivalent to the following equations in well-defined products, expanded and with known terms cancelled out:

$$
\begin{aligned}
\left(e_{1}+e_{0}+e_{-1}\right) \bullet\left(e_{-2}-e_{-3}\right) & =-e_{-1}-e_{-2} \\
\left(e_{1}+e_{0}+e_{-1}\right) \bullet\left(e_{3}+e_{2}\right) & =e_{2}-e_{1} \\
\left(e_{2}+e_{0}+e_{-2}\right) \bullet\left(e_{3}-e_{1}\right) & =-e_{3}-e_{-1} \\
\left(e_{2}+e_{0}+e_{-2}\right) \bullet\left(e_{-1}+e_{-3}\right) & =e_{1}-e_{-3} \\
\left(e_{3}+e_{0}+e_{-3}\right) \bullet\left(e_{2}-e_{-1}\right) & =e_{3}+e_{-2} \\
\left(e_{3}+e_{0}+e_{-3}\right) \bullet\left(e_{1}+e_{-2}\right) & =-e_{2}+e_{-3} \\
\left(e_{3}+e_{-2}+e_{-1}\right) \bullet\left(e_{2}+e_{1}+e_{-3}\right) & =e_{3}-e_{2}-e_{1}+e_{-1}+e_{-2}-e_{-3}
\end{aligned}
$$

In order to assist with calculations, I include a multiplication table for this product here. In this table, blank entries may be taken to be zero, since in any permissible calculation they will always cancel out. The terms $e_{i} \bullet e_{-i}$ may be taken to be $\pm e_{0}$ in various ways. One choice is given in the table.

\begin{tabular}{c|ccccccc|}
$\bullet$ & $e_{-3}$ & $e_{-2}$ & $e_{-1}$ & $e_{0}$ & $e_{1}$ & $e_{2}$ & $e_{3}$ \\
\hline$e_{-3}$ & & $e_{-3}$ & $-e_{-2}$ & & & & $-e_{0}$ \\
$e_{-2}$ & $-e_{-3}$ & & & & $e_{-1}$ & $-e_{0}$ & \\
$e_{-1}$ & $e_{-2}$ & & & & & $-e_{1}$ & \\
$e_{0}$ & & & & & & & \\
$e_{1}$ & & $-e_{-1}$ & & & & & $e_{2}$ \\
$e_{2}$ & & $e_{0}$ & $e_{1}$ & & & & $-e_{3}$ \\
$e_{3}$ & $e_{0}$ & & & & $-e_{2}$ & $e_{3}$ & \\
\hline
\end{tabular}

Indeed, the non-zero values of this product are best summarised in the following table.

\begin{tabular}{c|ccc|}
$\bullet$ & $e_{-3}$ & $e_{1}$ & $e_{2}$ \\
\hline$e_{-2}$ & $-e_{-3}$ & $e_{-1}$ & $-e_{0}$ \\
$e_{-1}$ & $e_{-2}$ & 0 & $-e_{1}$ \\
$e_{3}$ & $e_{0}$ & $-e_{2}$ & $e_{3}$ \\
\hline
\end{tabular}

Let $\mathbb{W}$ denote the algebraic structure consisting of the vector space $W$ endowed with the bilinear form $b$, the trilinear form $t$, and the partial product $\bullet$. Let $R(q)$ be the automorphism group of $\mathbb{W}$, that is, the group of linear maps on $W$ which preserve $b, t$ and $\bullet$. The geometry $\mathcal{G}$ on which it acts consists of the points $\langle v\rangle$ defined by the property that $v=v \bullet w$ for some $w$. (If there is danger of confusion, I will call these points bullet points, since they are defined by the bullet product.) The main result of this paper is that $R(q)$ is isomorphic to the 
Ree group as usually defined. More specifically, our results are summarised in the following theorem.

Theorem 1. 1. The geometry $\mathcal{G}$ consists of $q^{3}+1$ points.

2. The group $R(q)$ acts 2-transitively on the points of $\mathcal{G}$.

3. $R(q)$ has order $q^{3}\left(q^{3}+1\right)(q-1)$.

4. $R(q)$ permutes the $q^{6}+q^{3}$ vectors of norm 1 in $W$ transitively.

5. The centraliser of an involution in $R(q)$ is $2 \times \mathrm{PSL}_{2}(q)$.

6. The Sylow 2-normaliser in $R(q)$ is $2^{3}: 7: 3$.

7. The normaliser of a $2^{2}$ in $R(q)$ is $\left(2^{2} \times D_{(q+1) / 2}\right): 3$.

8. If $q>3$ then $R(q)$ is simple.

9. $R(3) \cong \mathrm{PSL}_{2}(8): 3$.

10. $R(q)$ is isomorphic to the Ree group as usually defined.

The rest of this paper is devoted to proving these facts.

\section{Proofs}

We have already observed that the forms $b$ and $t$ (and therefore the octonion product $*$ ) respect the grading of the coordinates, in the sense that $b\left(e_{i}, e_{j}\right)=0$ unless $i+j=0$, and $t\left(e_{i}, e_{j}, e_{k}\right)=0$ unless $i+j+k=0$. The product $\bullet$ also respects the grading in the sense that (whenever $e_{i} \bullet e_{j}$ is defined and non-zero) $e_{i} \bullet e_{j} \in\left\langle e_{g(i+j)}\right\rangle$, where $g$ is the monotonic function taking $-5,-4,-1,0,1,4,5$ to $-3,-2,-1,0,1,2,3$ respectively. This means that it makes sense to talk about the leading term of a vector $v=\sum_{i=-3}^{3} \lambda_{i} e_{i}$, that is the term $e_{i}$ for the maximum value of $i$ for which $\lambda_{i} \neq 0$. If $u \bullet v=w$ then without loss of generality the leading terms of $u$ and $v$ are different, and the leading term of $w$ is equal to the product of the leading terms of $u$ and $v$. In particular, if $v \bullet w=v$, then the leading term of $v$ occurs on both sides of one of the defining equations $e_{i} \bullet e_{j}= \pm e_{k}$, so by inspection is either $e_{-3}$ or $e_{3}$.

Next we investigate the stabiliser of $\left\langle e_{0}\right\rangle$.

Lemma 1. The stabiliser in $R(q)$ of $\left\langle e_{0}\right\rangle$ is a dihedral group of order $2(q-1)$, generated by the diagonal elements

$$
d(\lambda)=\operatorname{diag}\left(\lambda^{-3^{n+1}-2}, \lambda^{-3^{n+1}-1}, \lambda^{-1}, 1, \lambda, \lambda^{3^{n+1}+1}, \lambda^{3^{n+1}+2}\right)
$$

and the involution $r: e_{0} \mapsto-e_{0}, e_{i} \mapsto e_{-i}$ for $i \neq 0$.

Proof. Observe that the map $v \mapsto v * e_{0}$ is diagonalisable, and has three eigenvalues $0,-1$, and 1 , with corresponding eigenspaces $V_{0}=\left\langle e_{0}\right\rangle, V_{-1}=\left\langle e_{-3}, e_{1}, e_{2}\right\rangle$ and $V_{1}=\left\langle e_{3}, e_{-1}, e_{-2}\right\rangle$. Now $V_{-1}$ contains a unique (bullet) point, namely $\left\langle e_{-3}\right\rangle$, since any bullet point in $V_{-1}$ has leading term $e_{-3}$. Therefore by symmetry $V_{1}$ contains a unique point, namely $\left\langle e_{3}\right\rangle$. In particular, the stabiliser of the vector $e_{0}$ fixes the 1-spaces $\left\langle e_{-3}\right\rangle$ and $\left\langle e_{3}\right\rangle$, so also fixes the spaces $V_{1} \cap\left(e_{-3} \bullet W\right)=\left\langle e_{-2}\right\rangle$ and similarly $\left\langle e_{2}\right\rangle$. Therefore it also fixes $V_{1} \cap\left(e_{-2} \bullet W\right)=\left\langle e_{-1}\right\rangle$ and similarly $\left\langle e_{1}\right\rangle$, so acts diagonally on the given basis. 
Now any diagonal element $e_{i} \mapsto \lambda_{i} e_{i}$ which preserves $b$ has $\lambda_{-i}=\lambda_{i}{ }^{-1}$. If it also preserves $t$, then $\lambda_{i} \lambda_{0} \lambda_{-i}=1$ and therefore $\lambda_{0}=1$; and $\lambda_{1} \lambda_{2}=\lambda_{3}$. To preserve $\bullet$ it must also satisfy $\left(\lambda_{2} \lambda_{-1}\right)^{3^{n}}=\lambda_{1}$, that is $\lambda_{2}=\lambda_{1}{ }^{3^{n+1}+1}$. Conversely, it is easy to check that if these equations hold then the given diagonal element preserves $b, t$ and $\bullet$. These elements form a cyclic group of order $q-1$, which is therefore the stabiliser of $e_{0}$. Consequently, the stabiliser of $\left\langle e_{0}\right\rangle$ is a dihedral group of order $2(q-1)$.

Next we study the stabiliser, $B$ say, of $\left\langle e_{-3}\right\rangle$. Notice first that if $v$ is any isotropic vector in $V_{1}$, then $v \bullet W=v \bullet V_{-1}=v \bullet\left(V_{-1} \cap v^{\perp}\right)$ and is therefore spanned by two vectors $v \bullet w_{1}$ and $v \bullet w_{2}$, where $w_{1}, w_{2}$ span the 2 -space $V_{-1} \cap v^{\perp}$. Similarly for $v \in V_{-1}$, and $\left\langle w_{1}, w_{2}\right\rangle=V_{1} \cap v^{\perp}$. Now we see that the group $B$ must fix $e_{-3} \bullet W=\left\langle e_{-3} \bullet e_{-2}, e_{-3} \bullet e_{-1}\right\rangle=\left\langle e_{-3}, e_{-2}\right\rangle$, and therefore also fixes $\left\langle e_{-3}, e_{-2}\right\rangle \bullet W=\left\langle e_{-3} \bullet e_{-2}, e_{-3} \bullet e_{-1}, e_{-2} \bullet e_{1}\right\rangle=\left\langle e_{-3}, e_{-2}, e_{-1}\right\rangle$. It also fixes their orthogonal complements, and therefore acts as lower triangular matrices with respect to the given ordered basis. In particular, $\left\langle e_{0}\right\rangle$ maps to one of at most $q^{3}$ possible 1-spaces, so by Lemma 1 the stabiliser of $\left\langle e_{-3}\right\rangle$ has order at most $q^{3}(q-1)$. We shall show that the stabiliser has exactly this order by exhibiting symmetries which map $e_{0}$ to $e_{0}+e_{-1}$, or $e_{0}+e_{-2}$, or $e_{0}+e_{-3}$. Indeed the method used in Lemma 1 to calculate the stabiliser of $e_{0}$ enables us to complete any map of $e_{0}$ to $e_{0}+$ (lower terms) to a unique map on the whole space.

Lemma 2. The stabiliser of $e_{-3}$ in $R(q)$ is transitive on the $q^{3}$ vectors of shape $e_{0}+\lambda_{-1} e_{-1}+\lambda_{-2} e_{-2}+\lambda_{-3} e_{-3}$.

Proof. Consider first the most difficult case, $e_{0} \mapsto e_{0}+e_{-1}$, and write $e_{i}^{\prime}$ for the image of $e_{i}$ under this map. We assume that $e_{-3}$ is fixed, and then calculate the images of the remaining basis vectors $e_{-2}, e_{-1}, e_{1}, e_{2}, e_{3}$ in that order. The eigenspaces of octonion multiplication by $e_{0}^{\prime}=e_{0}+e_{-1}$ are $V_{0}^{\prime}=\left\langle e_{0}+e_{-1}\right\rangle$, $V_{1}^{\prime}=\left\langle e_{3}-e_{2}, e_{-1}, e_{-2}+e_{-3}\right\rangle$ and $V_{-1}^{\prime}=\left\langle e_{-3}, e_{2}, e_{1}-e_{0}+e_{-1}\right\rangle$. Since $e_{-3}$ is fixed, $e_{-2}^{\prime}$ lies in $\left(e_{-3} \bullet W\right) \cap V_{1}^{\prime}=\left\langle e_{-2}+e_{-3}\right\rangle$. Also, $e_{-3}^{\prime} \bullet e_{-2}^{\prime}=e_{-3}^{\prime}$, so $e_{-2}^{\prime}=e_{-2}+e_{-3}$. Then

$$
\begin{aligned}
\left(e_{-2}+e_{-3}\right) \bullet W & =\left(e_{-2}+e_{-3}\right) \bullet\left\langle e_{-3}, e_{1}-e_{0}+e_{-1}\right\rangle \\
& =\left\langle e_{-3}, e_{-1}-e_{-2}\right\rangle
\end{aligned}
$$

which intersects $V_{1}^{\prime}$ in $\left\langle e_{-1}-e_{-2}-e_{-3}\right\rangle$, and since $e_{-1}^{\prime} \bullet e_{-3}^{\prime}=e_{-2}^{\prime}$ we have $e_{-1}^{\prime}=e_{-1}-e_{-2}-e_{-3}$. Next

$$
\begin{aligned}
\left(e_{-1}-e_{-2}-e_{-3}\right) \bullet W= & \left(e_{-1}-e_{-2}-e_{-3}\right) \bullet\left\langle e_{-3}, e_{2}+e_{1}-e_{0}+e_{-1}\right\rangle \\
= & \left\langle e_{-2}+e_{-3},-e_{1}-e_{-1}+e_{0}+e_{-2}\right\rangle
\end{aligned}
$$

which intersects $V_{-1}^{\prime}$ in $\left\langle e_{1}-e_{0}+e_{-1}+e_{-3}\right\rangle$, and the correct scalar multiple is determined by the requirement that $e_{-2}^{\prime} \bullet e_{1}^{\prime}=e_{-1}^{\prime}$. Then

$$
\begin{aligned}
\left(e_{1}-e_{0}+e_{-1}+e_{-3}\right) \bullet W & =\left(e_{1}-e_{0}+e_{-1}+e_{-3}\right) \bullet\left\langle e_{-2}+e_{-3}, e_{3}-e_{2}-e_{-1}\right\rangle \\
& =\left\langle-e_{-1}+e_{-2}+e_{-3}, e_{2}+e_{1}-e_{0}+e_{-2}\right\rangle
\end{aligned}
$$


which intersects $V_{-1}^{\prime}$ in $\left\langle e_{2}+e_{1}-e_{0}+e_{-1}-e_{-3}\right\rangle$, and the correct scalar multiple is given by $e_{2}^{\prime} \bullet e_{-1}^{\prime}=e_{1}^{\prime}$. This gives us all but the last row of the matrix, which is then determined by orthogonality, as follows:

$$
\left(\begin{array}{cccccccc}
1 & & & & & & \\
1 & 1 & & & & & \\
-1 & -1 & 1 & & & & \\
0 & 0 & 1 & 1 & & & \\
1 & 0 & 1 & -1 & 1 & & \\
-1 & 0 & 1 & -1 & 1 & 1 & \\
-1 & -1 & -1 & 0 & 0 & -1 & 1
\end{array}\right) .
$$

Conversely, we need to check that this matrix preserves the algebraic structure. To check that it preserves $b$, we easily check that $V_{0}^{\prime}$ is orthogonal to $V_{1}^{\prime}$ and $V_{-1}^{\prime}$, and that $V_{1}^{\prime}$ and $V_{-1}^{\prime}$ are totally isotropic. Finally, the bases $\left\{e_{-2}^{\prime}, e_{-1}^{\prime}, e_{3}^{\prime}\right\}$ for $V_{1}^{\prime}$ and $\left\{e_{2}^{\prime}, e_{1}^{\prime}, e_{-3}^{\prime}\right\}$ for $V_{-1}^{\prime}$ are easily checked to be dual to each other.

To check that the trilinear form $t$ (or, equivalently, the octonion product $*$ ) is preserved, notice that by construction the products with $e_{0}^{\prime}$ are correct. This deals with all terms of the trilinear form involving $e_{0}^{\prime}$. Every other term involves either two vectors from $V_{1}^{\prime}$ or two vectors from $V_{-1}^{\prime}$, so it suffices to consider products of such pairs of vectors. Now we calculate

$$
\begin{aligned}
& e_{-2}^{\prime} * e_{-1}^{\prime}=\left(e_{-2}+e_{-3}\right) *\left(e_{-1}-e_{-2}-e_{-3}\right) \\
&=\left(e_{-2}+e_{-3}\right) * e_{-1}=-e_{-3}=-e_{-3}^{\prime} \\
& e_{-2}^{\prime} * e_{3}^{\prime}=\left(e_{-2}+e_{-3}\right) *\left(e_{3}-e_{2}-e_{-1}-e_{-2}-e_{-3}\right) \\
&=e_{1}-e_{0}+e_{-1}+e_{-3}=e_{1}^{\prime} \\
& e_{-1}^{\prime} * e_{3}^{\prime}=\left(e_{-1}-e_{-2}-e_{-3}\right) *\left(e_{3}-e_{2}-e_{-1}-e_{-2}-e_{-3}\right) \\
&=-e_{2}-e_{1}+e_{0}-e_{-1}+e_{-3}=-e_{2}^{\prime} \\
& e_{-3}^{\prime} * e_{1}^{\prime}=e_{-3} *\left(e_{1}-e_{0}+e_{-1}+e_{-3}\right)=e_{-2}+e_{-3}=e_{-2}^{\prime} \\
& e_{-3}^{\prime} * e_{2}^{\prime}=e_{-3} *\left(e_{2}+e_{1}-e_{0}+e_{-1}-e_{-3}\right)=-e_{-1}+e_{-2}-e_{-3}=-e_{-1}^{\prime} \\
& e_{1}^{\prime} * e_{2}^{\prime}=\left(e_{1}-e_{0}+e_{-1}+e_{-3}\right) *\left(e_{2}+e_{1}-e_{0}+e_{-1}-e_{-3}\right) \\
&=e_{3}+e_{1}+e_{0}+e_{-2}-e_{2}-e_{1}+e_{-1}+e_{-3}-e_{0}-e_{-1} \\
& \quad-e_{-1}+e_{-2}+e_{-3}=e_{3}^{\prime}
\end{aligned}
$$

which by the cyclic symmetry of $t$ is enough.

Checking the bullet product is a little more time-consuming, as we have to check the 14 defining equations individually. The first six are as follows.

$$
\begin{aligned}
e_{-3}^{\prime} \bullet e_{-2}^{\prime} & =e_{-3} \bullet\left(e_{-2}+e_{-3}\right)=e_{-3}=e_{-3}^{\prime} \\
e_{-1}^{\prime} \bullet e_{-3}^{\prime} & =\left(e_{-1}-e_{-2}-e_{-3}\right) \bullet e_{-3}=e_{-2}+e_{-3}=e_{-2}^{\prime} \\
e_{-2}^{\prime} \bullet e_{1}^{\prime} & =\left(e_{-2}+e_{-3}\right)\left(e_{1}-e_{0}+e_{-1}+e_{-3}\right)=e_{-1}-e_{-3}-e_{-2}=e_{-1}^{\prime} \\
e_{2}^{\prime} \bullet e_{-1}^{\prime} & =\left(e_{2}+e_{1}-e_{0}+e_{-1}-e_{-3}\right) \bullet\left(e_{-1}-e_{-2}-e_{-3}\right) \\
& =e_{1}-e_{0}+e_{-1}+e_{-2}+e_{-3}-e_{-2}=e_{1}^{\prime} \\
e_{1}^{\prime} \bullet e_{3}^{\prime} & =\left(e_{1}-e_{0}+e_{-1}+e_{-3}\right) \bullet\left(e_{3}-e_{2}-e_{-1}-e_{-2}-e_{-3}\right) \\
& =-e_{0}+e_{2}+e_{-2}+e_{-1}-e_{-3}+e_{1}-e_{-2}=e_{2}^{\prime} \\
e_{3}^{\prime} \bullet e_{2}^{\prime} & =\left(e_{3}-e_{2}-e_{-1}-e_{-2}-e_{-3}\right) \bullet\left(e_{2}+e_{1}-e_{0}+e_{-1}-e_{-3}\right) \\
& =e_{3}-e_{2}+e_{1}-e_{-1}+e_{-2}-e_{-3}-e_{1}+e_{-2}=e_{3}^{\prime}
\end{aligned}
$$


The other eight calculations are similar and left as an exercise for the reader.

Similar calculations produce symmetries which extend the maps $e_{0} \mapsto e_{0}+e_{-2}$ and $e_{0} \mapsto e_{0}+e_{-3}$. The resulting matrices are

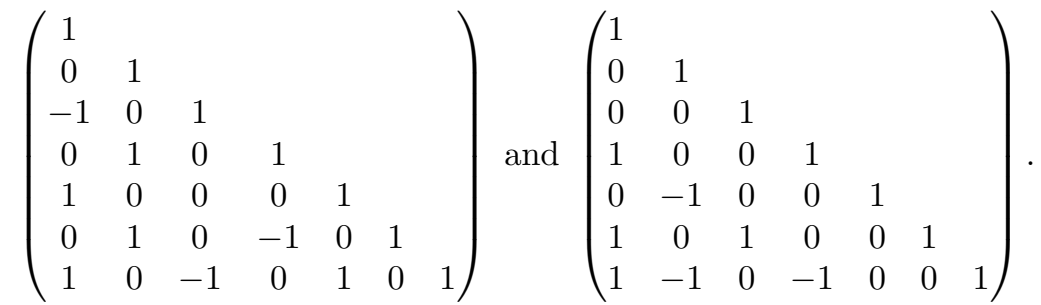

Conjugating these and their inverses by suitable diagonal elements, and multiplying them together, gives the general case.

It follows immediately that the stabiliser of $e_{-3}$ has order $q^{3}$, and the stabiliser of the bullet point $\left\langle e_{-3}\right\rangle$ has order $q^{3}(q-1)$. We next show that there are exactly $q^{3}+1$ bullet points.

Lemma 3. The number of (bullet) points is $q^{3}+1$, and $R(q)$ permutes them transitively.

Proof. Observe from the multiplication table that if $v$ and $w$ are isotropic vectors with octonion product $v * w=0$, then $v$ and $w$ are mutually orthogonal. Suppose now that $\langle v\rangle$ is a point with leading term $e_{3}$, so that $v=v \bullet w$ and $w$ has leading term $e_{2}$. Then from the proof of the previous lemma we know that for every $\lambda \in \mathbb{F}_{q}$ there is an element of $R(q)$ mapping $e_{3} \mapsto e_{3}+\lambda e_{2}$ so we may assume that $v$ has no term in $e_{2}$. Similarly, applying a suitable symmetry $e_{3} \mapsto e_{3}+\lambda e_{1}$ we may assume $v$ has no term in $e_{1}$, and then we may apply a symmetry $e_{3} \mapsto e_{3}+\lambda e_{0}$ to remove the term in $e_{0}$. Therefore $v=e_{3}+\lambda_{-1} e_{-1}+\lambda_{-2} e_{-2}+\lambda_{-3} e_{-3}$. But $v$ is isotropic, so $\lambda_{-3}=0$. Now applying the symmetry $r: e_{i} \mapsto e_{-i}, e_{0} \mapsto-e_{0}$ we obtain a point whose leading term is not $e_{3}$. This point must be $\left\langle e_{-3}\right\rangle$, so $v=e_{3}$. Therefore every point is either $\left\langle e_{-3}\right\rangle$, or one of the $q^{3}$ images of $\left\langle e_{3}\right\rangle$ under the action of the stabiliser of $\left\langle e_{-3}\right\rangle$.

This proves the first part of the main theorem. Since the stabiliser of the two points $\left\langle e_{-3}\right\rangle$ and $\left\langle e_{3}\right\rangle$ has order $q-1$, the point stabiliser (of order $q^{3}(q-1)$ ) must be transitive on the remaining $q^{3}$ points. Hence $R(q)$ acts 2 -transitively on $\mathcal{G}$, proving the second part of the theorem. It follows immediately that the order of $R(q)$ is $q^{3}\left(q^{3}+1\right)(q-1)$, which is the third part of the theorem. Since the stabiliser of the vector $e_{0}$ of norm 1 has order $q-1$, it follows that $R(q)$ is transitive on the $q^{6}+q^{3}$ vectors of norm 1 in $W$, which is the fourth part of the theorem.

Since $q \equiv 3 \bmod 8$, we have $q^{3}+1 \equiv 4 \bmod 8$ and $q-1 \equiv 2 \bmod 4$ and therefore the Sylow 2-subgroup of $R(q)$ has order 8 . Next consider the centraliser of the involution $d(-1)=\operatorname{diag}(-1,1,-1,1,-1,1,-1)$. This involution fixes precisely $q+1$ points, namely $\left\langle e_{-3}\right\rangle$ and the $q$ images of $\left\langle e_{3}\right\rangle$ under the maps defined by 
$e_{-3} \mapsto e_{-3}, e_{0} \mapsto e_{0}+\lambda e_{-2}$. Moreover its centraliser in the point stabiliser is a group of order $q(q-1)$ and shape $2 \times 3^{2 n+1}: C_{q-1}$. Therefore its centraliser in $R(q)$ has order $q(q-1)(q+1)$. Now on the fixed 3 -space $\left\langle e_{-2}, e_{0}, e_{2}\right\rangle$ there are elements acting as

$$
\left(\begin{array}{ccc}
1 & 0 & 0 \\
1 & 1 & 0 \\
1 & -1 & 1
\end{array}\right),\left(\begin{array}{ccc}
\lambda^{2} & 0 & 0 \\
0 & 1 & 1 \\
0 & 0 & \lambda^{-2}
\end{array}\right) \text { and }\left(\begin{array}{ccc}
0 & 0 & 1 \\
0 & -1 & 0 \\
1 & 0 & 0
\end{array}\right)
$$

where $\lambda \in \mathbb{F}_{q}$. These matrices generate $\Omega_{3}(q) \cong \operatorname{PSL}_{2}(q)$. Since $R(q)$ contains an elementary abelian group of order 4 , while $\mathrm{SL}_{2}(q)$ does not, it follows that the centraliser of the given involution is $2 \times \operatorname{PSL}_{2}(q)$. This is the fifth part of the theorem.

In particular, the Sylow 2-subgroup is elementary abelian of order 8 , and its normaliser contains $2 \times A_{4}$. The latter group contains three classes of involutions, and it is easy to check that each involution fixes at least one point. Specifically, the element $r$ fixes the point $e_{3}-e_{1}-e_{0}+e_{-1}-e_{-3}$, while $d(-1) r$ fixes the point $e_{3}-e_{2}+e_{1}+e_{0}+e_{-1}+e_{-2}+e_{-3}$. But all involutions which fix a point are conjugate. Therefore all involutions in $R(q)$ are conjugate, and the Sylow 2normaliser is transitive on its non-trivial elements, so has order 168 and shape $2^{3}: 7: 3$. This is the sixth part of the theorem. The seventh part follows immediately from the fact that the centraliser of a $2^{2}$ is $2 \times D_{q+1}$, inside $2 \times \operatorname{PSL}_{2}(q)$.

Simplicity of $R(q)$ for $q>3$ follows from Iwasawa's Lemma as in [7]. For completeness we give the proof again here. We have shown that $R(q)$ acts faithfully and 2-transitively (therefore primitively) on a set of $q^{3}+1$ points. The point stabiliser is a soluble group of order $q^{3}(q-1)$. Provided $q>3$, the point stabiliser is generated by the elements of order $q-1$, and therefore so is $R(q)$. Each such element is the product of an involution and an element of odd order $(q-1) / 2$. The involutions are commutators already in $2^{3}: 7: 3$, and the elements of order $(q-1) / 2$ are commutators inside $D_{2(q-1)}$. Hence $R(q)$ is perfect (when $q>3$ ), and by Iwasawa's Lemma is simple.

In the case $q=3$, the Sylow 2-normaliser $2^{3}: 7: 3$ has index 9 in $R(q)$, so $R(q)$ embeds in $S_{9}$ as a 3 -transitive group on 9 points. Therefore $R(3) \cong \mathrm{PSL}_{2}(8): 3$, which is the ninth part of the theorem. To see that our groups $R(q)$ are isomorphic to the Ree groups as traditionally defined, we could invoke the Classification Theorem for Finite Simple Groups, or more specifically the classification of simple groups with involution centraliser $2 \times \mathrm{PSL}_{2}\left(3^{2 n+1}\right)$ (see [1]). Perhaps more satisfactorily, one can calculate explicit formulae for the $q^{3}+1$ points and observe that they are essentially the same as those calculated by Tits [5] (see also [6]). Nevertheless, this is not a particularly easy calculation to do, and an alternative is to observe that our matrix generators are the same (up to some sign-changes on the basis vectors) as those calculated from the Lie theory by several people, including Howlett, Rylands and Taylor [2]. 


\section{Further remarks}

The definitions of both the octonion product $*$ and the partial product $\bullet$ can be motivated by consideration of the root system of type $G_{2}$.

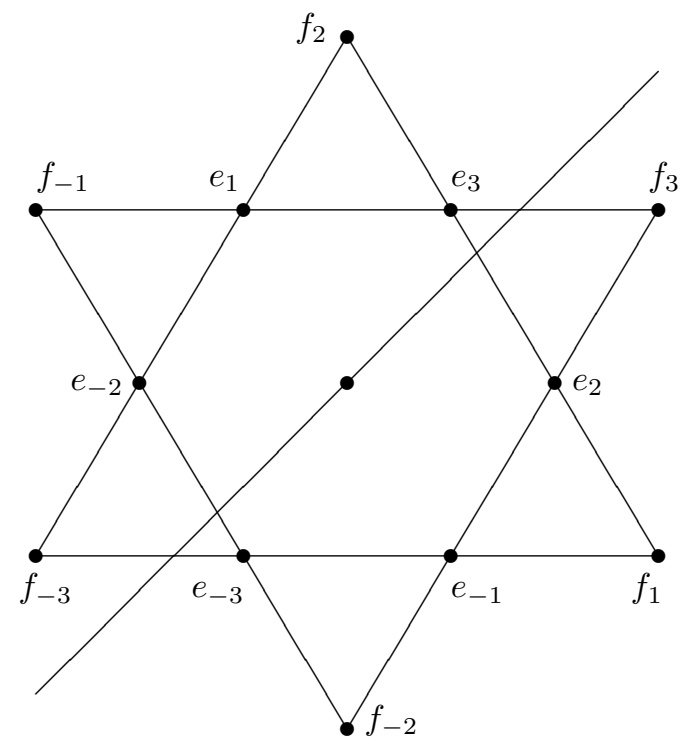

In this picture, if $e_{i}$ and $e_{j}$ are short roots whose sum is a short root $e_{k}$, and $e_{j}$ is reached from $e_{i}$ by turning clockwise through $1 / 3$ of a rotation, then $e_{i} * e_{j}=e_{k}$. Similarly, if $e_{i}$ and $e_{j}$ are short roots whose sum is a long root $f_{k}$, and $e_{j}$ is reached from $e_{i}$ by turning clockwise through $1 / 6$ of a rotation, then $e_{i} \bullet e_{j}=e_{k}$. The map between the short and long roots is given by reflection in the line at $\pi / 4$ to the vertical, followed by the appropriate scaling. The grading of the coordinates is given by the ordering of their projections onto this line.

If we relabel the roots by complex numbers, so that $e_{1}$ becomes $e_{\omega}$ and $e_{-3}$ becomes $e_{\bar{\omega}}$ while $e_{2}$ becomes $e_{1}$, then we can write the above rules as $e_{\bar{\omega} z} * e_{\omega z}=$ $e_{-z}$ and $e_{\omega z} \bullet e_{-\bar{\omega} z}=e_{\bar{z}}$.

The construction works equally well for infinite fields, provided there is an automorphism $\sigma$ which squares to the inverse of the Frobenius automorphism. In particular, this requires the field to be perfect. By recasting the partial product as a coproduct (only defined modulo the octonion coproduct) we can remove the requirement for the field to be perfect, provided only that the Frobenius endomorphism has a square root.

It is easy to see that $R(q)$ has exactly three orbits on the $q^{6}-1$ isotropic vectors in $W$ : the orbit containing $e_{-3}$ has length $(q-1)\left(q^{3}+1\right)$, while the orbit containing $e_{-2}$ has length $(q-1) q\left(q^{3}+1\right)$ and the orbit containing $e_{-1}$ has length $(q-1) q^{2}\left(q^{3}+1\right)$. The $q^{6}-q^{3}$ vectors of norm -1 in fact fall into three orbits also, and the corresponding 1-space stabilisers are maximal (if $q>3$ ) subgroups of $R(q$ ) of shapes $\left(2^{2} \times D_{(q+1) / 2}\right): 3,(q+\sqrt{3 q}+1): 6$ and $(q-\sqrt{3 q}+1): 6$. The first of these 
is represented by the 1 -space $\left\langle e_{-2}+e_{2}\right\rangle$, whose stabiliser is the normaliser of the group $\langle r, d(-1)\rangle$.

\section{References}

[1] E. Bombieri, A. Odlyzko and D. Hunt, Thompson's problem $\left(\sigma^{2}=3\right)$, Invent. Math. 58 (1980), 77-100.

[2] R. B. Howlett, L. J. Rylands and D. E. Taylor, Matrix generators for exceptional groups of Lie type, J. Symb. Comput. 31 (2001), 429-445.

[3] R. Ree, A family of simple groups associated with the simple Lie algebra of type $\left(G_{2}\right)$, Amer. J. Math. 83 (1961), 432-462.

[4] R. Ree, A family of simple groups associated with the simple Lie algebra of type $\left(F_{4}\right)$, Bull. Amer. Math. Soc. 67 (1961), 115-116.

[5] J. Tits, Les groupes simples de Suzuki et Ree, Séminaire Bourbaki 6 (1960/1), exposé 210.

[6] H. van Maldeghem, Generalized polygons, Monographs in Mathematics, vol. 93 (Birkhäuser, 1998).

[7] R. A. Wilson, An elementary construction of the Ree groups of type ${ }^{2} G_{2}$, to appear in Proc. Edinburgh Math. Soc. (2010).

[8] R. A. Wilson, A simple construction of the Ree groups of type ${ }^{2} F_{4}$, J. Algebra 323 (2010), 1468-1481.

[9] R. A. Wilson, A new approach to the Suzuki groups, to appear in Math. Proc. Cambridge Philos. Soc..

Robert A. Wilson

School of Mathematical Sciences,

Queen Mary, University of London,

Mile End Road, London E1 4NS

e-mail: R.A.Wilson@qmul.ac.uk 